\title{
Test for a population expansion after a drastic reduction in population size using DNA sequence data
}

\author{
M Björklund \\ Department of Animal Ecology, Evolutionary Biology Centre, Uppsala University, Norbyvägen 18D, SE-752 36 Uppsala, Sweden
}

\begin{abstract}
Populations may, during their evolutionary history, go through drastic changes in population size due to bottlenecks or founder events upon colonization of new areas. This involves a subsample of haplotypes, causing the allele frequencies to be different from the original population. In addition, the period of recovery after a bottleneck can be of considerable length. If reproduction is unequal among individuals but random with regard to haplotype, large deviations from the patterns expected in a stable population may result. By means of computer simulation, I have analysed the patterns arising when populations undergo bottlenecks and then slowly recover, and used two new statistical tests for the detection of the bottleneck. A test
\end{abstract}

Keywords: bottleneck; population expansion; DNA sequences; statistical test

\section{Introduction}

The level of genetic variation in a population is determined by an interplay of a variety of factors such as mutation rate, effective population size, and selection and historic events, such as founder events and bottlenecks. It is well known that historic changes in population size can have profound effects on the patterns of genetic variation in a population, and, given the remarkable technical development of easy-to-use molecular techniques, a variety of methods have been developed to test the possible occurrence of a bottleneck (Cornuet and Luikart, 1996; Kimmel et al, 1998; Luikart and England, 1999; Reich et al, 1999; Garza and Williamson, 2001). All these methods were mainly designed for use with microsatellite data.

Although the use of microsatellites has immensely increased our understanding of past and current population genetic processes, there are drawbacks. First, there are still concerns about which microsatellite mutation model is the most accurate (Ellegren, 2000). Second, for most organisms, microsatellite primers are not available, and the development of new ones is time consuming and costly. On the other hand, general primers for certain sequence regions, such as the mitochondrial control region, are often available, or at

Received 25 February 2002; accepted 17 March 2003 based on the variance of the relative frequency of haplotypes had generally high power even at low sample size $(n=25)$. This statistic was most powerful after very strong bottlenecks and lost power with increasing propagule size. A test based on the variance of the pairwise differences shows slightly less power. As expected, power was reduced when migration into the founder population was allowed from the source population. This suggests that the test is particularly suited for detecting relatively recent and strong bottlenecks, and thus may be a valuable tool for identifying population events on a fine temporal scale, such as colonisations after the last glaciation.

Heredity (2003) 91, 481-486. doi:10.1038/sj.hdy.6800309 least quite easily modified to fit a particular organism. The development of statistical methods for analysing possible bottlenecks using DNA sequence data has lagged behind the development of other methods for analysing microsatellite data (but see Galtier et al, 2000).

Methods that have frequently been used to infer past bottlenecks include the mismatch distribution based on the sudden expansion model of Rogers and Harpending (1992), Rogers (1995) and Schneider and Excoffier (1999), and Tajima's D (Tajima, 1989a, b; Fu and Li, 1993a). However, these models are based on the assumption of an instantaneous increase of several orders of magnitude in population size, and thus, strictly speaking, test only if such an expansion has happened in the past and not if a bottleneck has actually occurred. Even though the simulations by Rogers and Harpending (1992) show that, under certain circumstances, the sudden expansion model may detect bottlenecks, the basic premise (a sudden large increase) is questionable from a biological point of view. The bottleneck and the subsequent recovery from it involve two major elements that affect genetic variance in subsequent generations: the sampling process and the variance in reproductive success of individuals. First, if sampling (assuming mtDNA) is random with regard to haplotypes, the remaining population may consist of a sample of the original population, and in particular many haplotypes are lost. Second, if individuals vary in reproductive success (which is highly likely) and if this variation is random with regard to haplotype, certain haplotypes will become over-represented in future generations. The importance 
of both these elements is dependent on the bottleneck size and the rate of increase during the recovery (Nei et al, 1975). If a population remains small for a long time after a bottleneck, genetic drift may heavily distort the distribution of alleles in the population. It is well known that the recovery after a bottleneck (such as after an invasion of a new habitat) can take a substantial number of generations (Elton, 1958). Thus, the elements described above are quite likely to be of great importance in natural populations.

In this paper, a biologically realistic stochastic model is presented, wherein a population drastically decreases in size and then recovers at a slow but realistic rate. This corresponds to situations where a large population undergoes a bottleneck for some reason (such as a disease) and then recovers, or when a small propagule disperses to a new habitat and then increases in the new habitat. The focus is the pattern of haplotype frequencies as a result of the sampling of a small number of individuals and the subsequent small population size. Next, a way of testing for the presence of a relatively recent strong bottleneck using DNA sequence data is devised, and finally the power of the new tests is tested.

\section{Methods}

In a situation where a population changes in size, either through a drastic reduction or where a small propagule enters a new geographical area, there are three factors that affect the distribution of haplotypes in a population. (i) The sampling event after which a random subsample of the original population remains. On average, this subsample should resemble the original population, but due to the stochastic nature of the sampling event a given subsample may differ considerably with regard to haplotype frequencies. (ii) When the population is still small, drift may drastically alter the frequency distribution. (iii) When the population slowly increases by chance, some haplotypes are more productive than others, which will augment the changes in frequencies that started with the sampling event and the subsequent drift. This will in turn lead to a frequency distribution of haplotypes that can differ considerably from the original and show peculiar patterns as a result of the three stochastic phases of the bottleneck and subsequent recovery.

Two simple ways of measuring this is to use the variance of the haplotype frequencies (hereafter called $\operatorname{Var}(S)$ since this is based on the number of segregation sites, $S)$ and the variance of the average number of pairwise differences $(=\operatorname{Var}(k))$. Since a restricted sample of individuals from any population will show patterns that may, to some extent, differ from that expected assuming an equilibrium population, the test will be performed by using the $\theta$-value estimated from the data and by coalescent simulation will create a null distribution to which the observed distributions will be compared.

\section{Computer simulation}

1. A large equilibrium (random mating, infinite sites) source population $(N=1000)$ was created by using the coalescent algorithm by Hudson (1990) with five different $\theta$-values $(0.1,0.5,1.0,3.0$ and 5.0).
2. From this large population, a sample of size $N_{0}(=20$ or 200) was used as a founder population.

3. This population was allowed to grow by allotting fitness values to each individual taken from a Poisson distribution with a mean scaled such that the population intrinsic rate of increase $(r)$ was either 0.009 or 0.09 . These values were chosen for two reasons: (1) It creates a situation where the phase of small population size is quite long so that the sampling process due to unequal reproduction can play a role. (2) These values are consistent with empirically estimated $r$-values for medium-sized mammals $(r=0.009)$ and many insects $(r=0.09)$ (Pianka, 1978). In other words, the fitness of different haplotypes was entirely determined by stochastic events. The generations were discrete. Migration was included by randomly drawing a number of individuals from the base population $(m=5)$ and adding them to the founder population from each generation. The procedure described above was then repeated. No mutations were allowed.

4. After the population had reached a size of 1000 individuals, a sample of $N_{s}=25$ individuals was taken. The population size was set to 1000 individuals because at that size and larger, genetic drift, which is the cause of the special patterns of haplotype relative frequency distribution after a bottleneck, has a very low impact. The populations reached the size 1000 at about 50 generations when $r=0.09$, and 200 generations when $r=0.009$.

5. The $\theta$-values used in the simulations were estimated from the data as $\theta=S / a_{1}$, where $a_{1}$ is

$$
a_{1}=\sum_{i=1}^{n-1} \frac{1}{i}
$$

and $n$ is the number of sequences (Watterson, 1975; Fu and $\mathrm{Li}, 1993 \mathrm{~b})$. The $\theta$-value was then used to create the expected values of $\operatorname{Var}(S)$ and $\operatorname{Var}(k)$ by taking 1000 samples of the same size (25) as above, generated by coalescent simulations (Hudson, 1990, see p. 1). This created a distribution of expected values and I compared the observed values with this distribution. If the observed value was smaller or larger than the $2.5 \%$ tails of the expected values, the test was scored as significant. Power was then estimated as the proportion of runs where a significant result was found, that is, the probability that a false null hypothesis is correctly rejected.

6. Power simulations were carried out by running 1000 replicates of each parameter combination using the test procedure described above (ie, each parameter combination involved 200000 simulations). Initially, 3000 replicates were used, but since the coefficient of variation (CV) of three replicates of 1000 each was only $0.5-3 \%$, the number of replicates was restricted to 1000 since the loss of accuracy was only minor. If a population went extinct, this run was redone. The simulations were carried out using Mathematica 4.1.

In summary, the tests were performed using five $\theta$-values $(0.1,0.5,1,3$ and 5), two migration rates, $m(0,5)$, two rates of increase, $r(0.009,0.09)$, and two founder population sizes, $N_{0}(20,200)$, creating in total 40 different parameter combinations. 


\section{Results}

\section{No migration}

Power defined as the probability of rejecting a null hypothesis of no bottleneck was generally very high for $\operatorname{Var}(S)$ and $\operatorname{Var}(k)$ when the increase in population size was slow $(r=0.009)$ and propagule size was low $\left(N_{0}=20\right)$, except when the diversity in the source population was very low $(\theta=0.1)$ (Figure 1a). However, even when $\theta$ was as low as 0.5 , power reached above $90 \%$. Power was reduced at low $\theta$-values when the increase in population size was fast $(r=0.09$, all else being equal) (Figure 1b). However, the lower power was only apparent when $\theta=0.1$, but reached $80 \%$ at $\theta=0.5$. When the founding population was large $\left(N_{0}=200\right)$, power was reduced in all cases (Figure 1c). Only when the increase in population size was slow $(r=0.009) \mathrm{did}$ power reach above $80 \%$ but only for $\operatorname{Var}(S)$ and with $\operatorname{Var}(k)$ being close to $80 \%$. When the rate of increase was higher $(r=0.09)$, none of the tests show a reasonable power (Figure 1d).

\section{Effect of migration}

As expected, when recurrent migration of five individuals from each generation was introduced, power dropped considerably. When the founding population was low $\left(N_{0}=20\right)$ power reached above $80 \%$ only for $\operatorname{Var}(S)$ and only when $\theta=5$, although at $\theta=3$ power was close to $80 \%$ (Figure 1e). The other test shows different patterns in relation to $\theta$, but in all cases shows a power of less than $50 \%$. When the rate of increase was high $(r=0.09)$, power was low in all cases, but reached above $70 \%$ for $\operatorname{Var}(S)$ when $\theta=3$ (Figure $1 \mathrm{f}$ ).

When the founding population was large $\left(N_{0}=200\right)$, power was generally low or very low for all tests. When the rate of increase was slow $(r=0.009)$ only $\operatorname{Var}(S)$ reached $70 \%$ when $\theta=5$, but in all other cases power was lower than 50\% (Figure $1 \mathrm{~g}$ ).

\section{Effect of migration, founding population size and sample size}

To analyse the effect of different numbers of migrants, a set of simulations with different numbers of migrants $(0$, 1,3 and 5) was run. Since the drop in power as a result of migration was most marked when $r=0.009, N_{0}=20$ and $\theta=0.5$, these values were used. As can be seen in Figure 2, power for $\operatorname{Var}(S)$ dropped almost linearly with increasing number of migrants. Already one migrant per generation causes power to drop just below 80\%, and when there were three migrants per generation power was as low as about $40 \%$.

Using the same setting but without migrants, the effect of founding population size was analysed. Power dropped with increasing propagule size (Figure 3), and when the propagule size reached 100, power of $\operatorname{Var}(S)$ dropped below $70 \%$.

The simulations in this study were all performed using a sample size of 25 since this is a common size of data sets currently gathered. To test the effect of a larger sample size, two additional simulations were made using the parameter values that were shown to have low power when sample size was 25. Thus, $r=0.009$, $N_{0}=200, \theta=0.5$ and $m=0$ and 5 were used. At a sample size of 25 and $m=0$ power was 0.38 for $\operatorname{Var}(S)$ (Figure 1c) and increased to 0.64 , and $\operatorname{Var}(k)$ increased from 0.32 to 0.53 . When $m=5$, power for $\operatorname{Var}(S)$ increased from 0.16 to 0.34 , and $\operatorname{Var}(k)$ increased in power from 0.21 to 0.34 . Thus, the effect of migration cannot easily be overcome by doubling the sample size.

A technical note: using $\operatorname{Var}(S)$ on a data set with 17 haplotypes and 10000 randomisations took about $11 \mathrm{~s}$ on a Pentium III machine using Mathematica 4.1. Thus, the test is very fast.
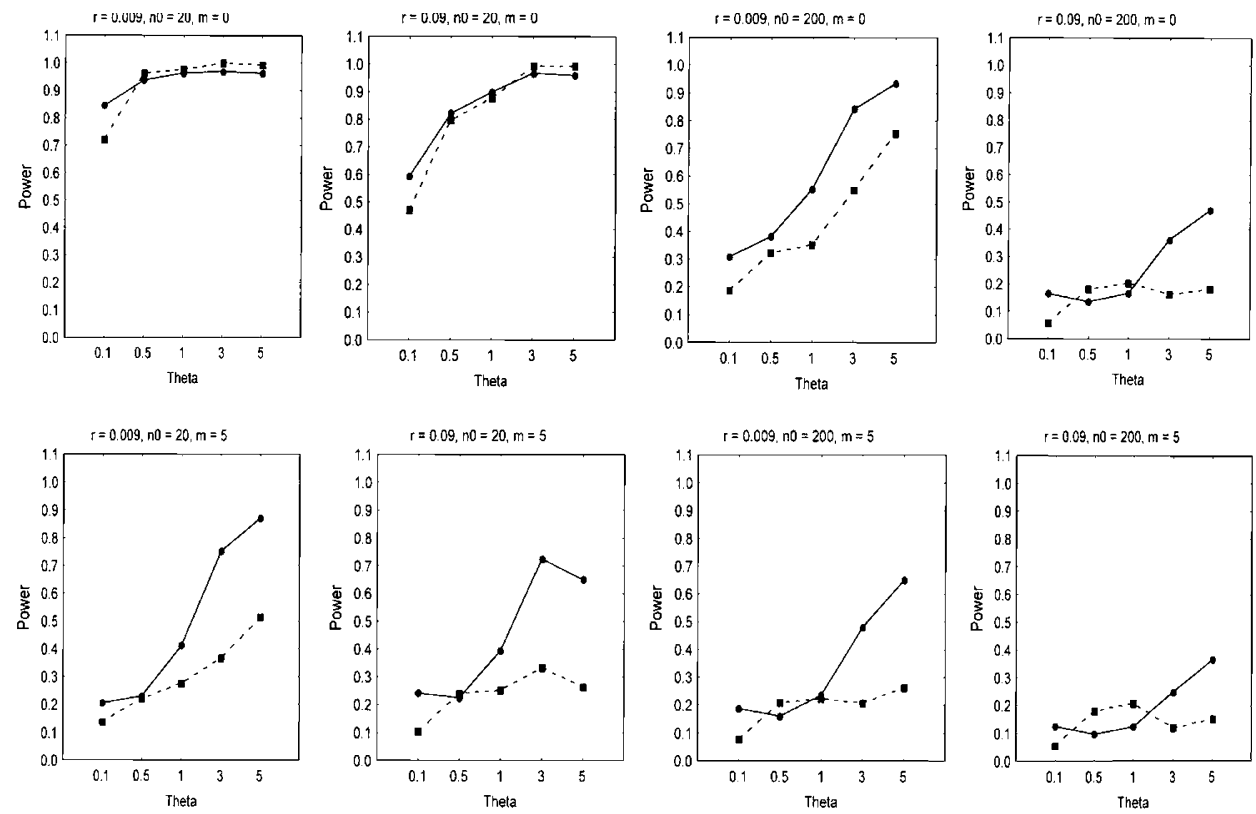

Figure 1 Power for two different tests for the presence of past bottlenecks for different intrinsic rates of increase $(r)$, propagule size $\left(N_{0}\right)$, initial diversity $(\theta)$ and number of migrants per generation from the base population. All simulations are based on a sample size of 25 individuals. Legends: $\operatorname{Var}(S)$ - unbroken line, $\operatorname{Var}(k)$ - broken line. 


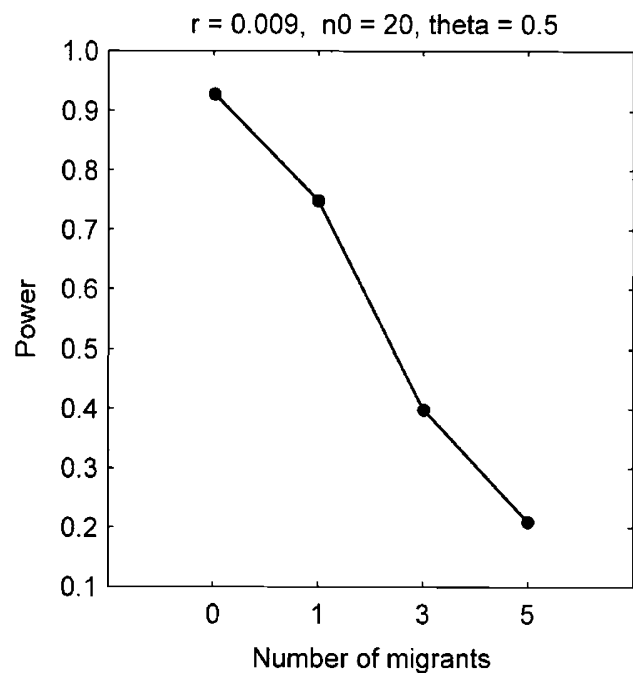

Figure 2 Effect of number of migrants on the power of $\operatorname{Var}(S)$ for a specific parameter combination. Each run is based on 1000 simulations.

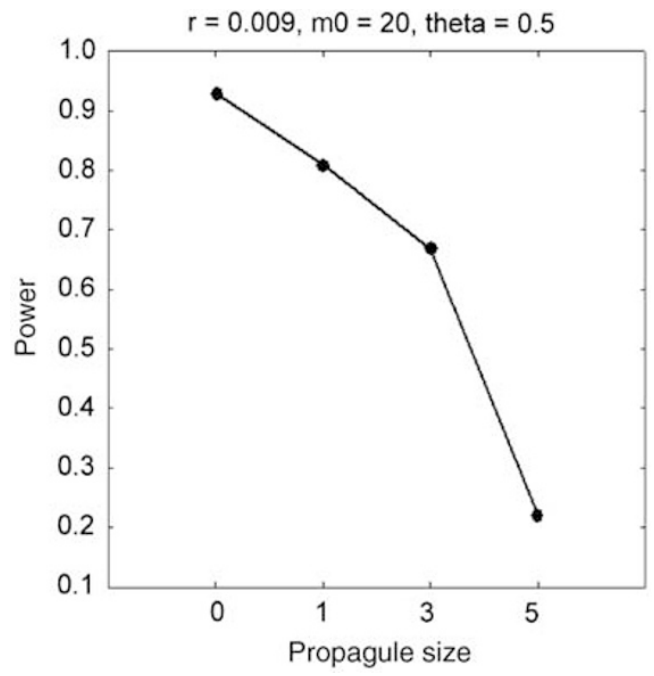

Figure 3 Effect of propagule size on power of $\operatorname{Var}(S)$ for a specific parameter combination. Each run is based on 1000 simulations.

\section{Examples}

There are several studies using mtDNA for the study of colonisation history and possible historical bottlenecks. The greenfinch was studied by Merilä et al (1997), where it was concluded that the colonisation process involved serial bottlenecks as shown by the low variability of the control region data. The authors did not test the expansion model, but found that the fit to the expansion model was in most cases better than the fit to an equilibrium model. Using Var $(S)$ instead, all but one population (Andujar) showed highly significant deviations ( $P$ ranging from 0.011 to less than 0.0001 ) from the expected variance in accordance with the bottleneck model presented above. To show the potential effect of time since the bottleneck, the data are presented in detail in Table 1, where the number of individuals sampled, the number of segregating sites, observed and expected Var (S) are shown as well as the $95 \%$ percentiles of the
Table 1 Results from the proposed bottleneck test on four populations of greenfinch

\begin{tabular}{lcccccc}
\hline Population & $\mathrm{N}$ & $\mathrm{S}$ & $\operatorname{Var}(\mathrm{S})$ & Mean & $95 \%$ & $\mathrm{P}$ \\
\hline Oulu & 14 & 3 & 0.21 & 0.0075 & $0.011-0.020$ & $<0.001$ \\
Kiev & 20 & 6 & 0.082 & 0.0039 & $0.00067-0.0093$ & $<0.001$ \\
Sevilla & 25 & 7 & 0.020 & 0.0034 & $0.0012-0.0079$ & $<0.001$ \\
Andujar & 17 & 3 & 0.015 & 0.0079 & $0.0010-0.021$ & 0.08
\end{tabular}

$N=$ number of individuals sampled, $S=$ number of segregating sites, $\operatorname{Var}(S)=$ variance of the haplotype frequency distribution, Mean= mean value of the simulated distribution, $95 \%=95$ percentiles of the simulated distribution, $P=$ probability that the observed value is within the simulated distribution assuming a normal distribution.

simulated distribution and $P$-value. The populations were chosen as to represent one very recent population $(<200$ generations) in the outmost part of the distribution (Oulu), one older population in the central part (Kiev) and two populations in the part of Europe where the species is assumed to have stayed during the last glaciation (Spain, Merilä et al, 1997). Clearly, the observed variance is far higher than the expected variance given the estimated $\theta$-value, except in Andujar, where the value is higher than the simulated mean but well within the $95 \%$ range. This suggests that the test can be used to test for bottlenecks at least several thousand generations back.

\section{Discussion}

The data presented show that of the two new tests for a bottleneck, the variance of the haplotype frequencies $(\operatorname{Var}(S))$ is the most powerful followed by the variance of the pairwise differences $(\operatorname{Var}(k))$. This was particularly so when there was a strong bottleneck $\left(N_{0}=20\right)$, and when the rate of rebound in population number was slow and with no migration from a source population. As migration increases, or $N_{0}$ increased, the power of the tests decreased substantially. This suggests that the tests proposed are indeed good tests for detecting strong bottlenecks as for example when new areas are colonised by a small number of founders.

The reason why $\operatorname{Var}(S)$ is so successful in detecting a bottleneck is straightforward. When a small subsample is drawn, only some haplotypes will be represented by chance. When the population increases in size, drift through unequal reproductive success will further augment the imbalance created by the original sampling. This will result in a pattern where a few haplotypes will predominate, and these haplotypes need not necessarily be the ones most common in the original population. This is illustrated in Figure 4, where four different runs are shown (parameter values given in the figure text). The hatched bars represent a subsample from the large source population (see Methods for details) that contains 15 haplotypes, while filled bars represent a subsample of the bottleneck population after it has reached 1000 individuals. Clearly, sometimes only one haplotype is present (Figure 4c) in the bottleneck population, while in others several haplotypes remain but in frequencies different from the source population. It is apparent that the number of haplotypes are fewer and occur in frequencies different from the source population. Thus, 

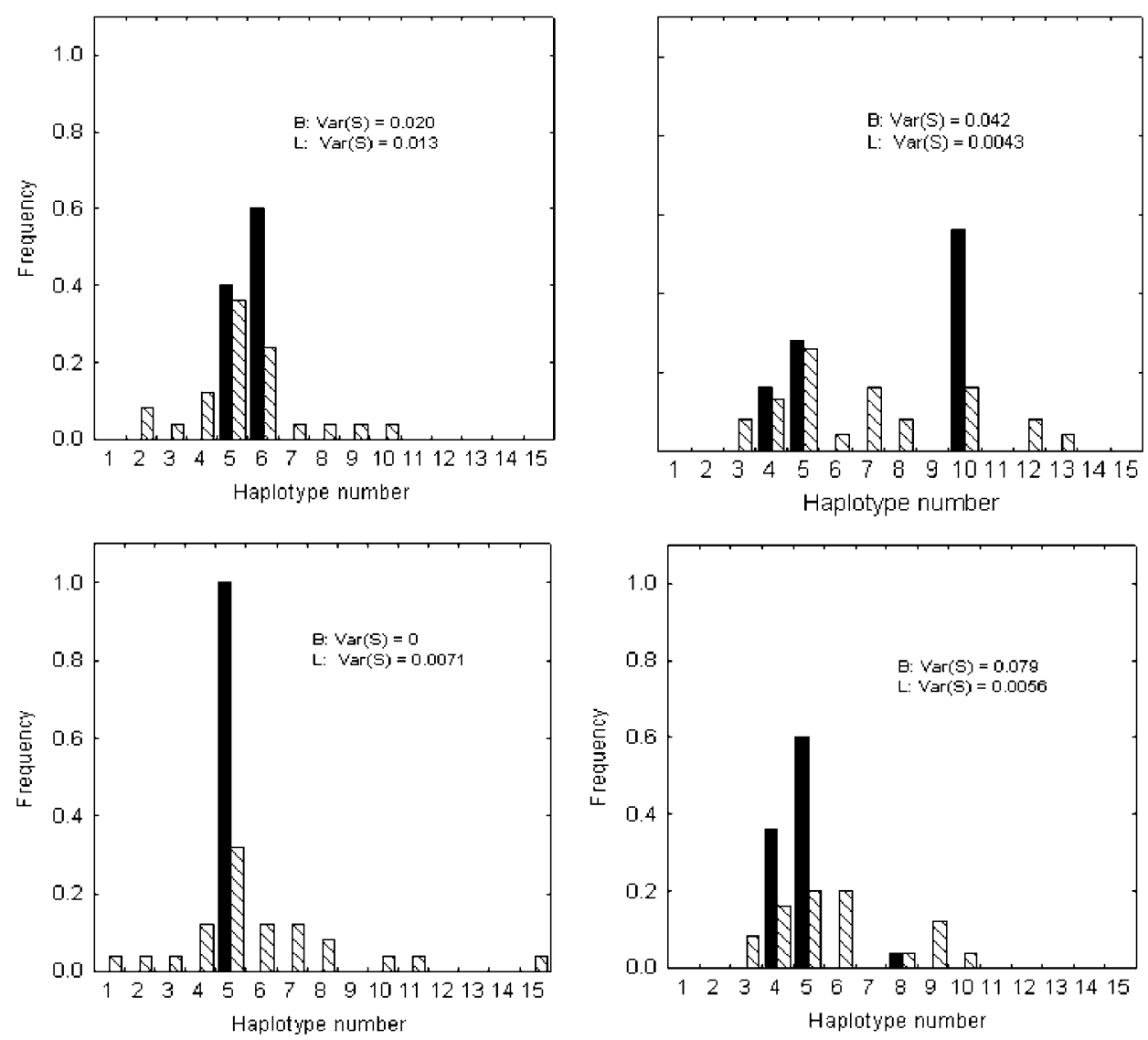

Figure 4 Effect of a bottleneck on the haplotype distribution. The original population contained 15 haplotypes. The hatched bars represent the sampling of 25 individuals from the original (L) population, while the filled bars represent the sampling of 25 individuals from a bottlenecked (B) population. The parameters were $\theta=1, r=0.009, m=0$ and $N_{0}=20$. The test statistic, $\operatorname{Var}(S)$, for the two samples are given in each figure.

when implementing the test, it is important to test for deviations from a simulated distribution in both tails.

It should be pointed out that all simulations presented in Figure 1 are based on a sample size of 25 individuals. This is a typical sample size in many studies, and apparently is enough to be able to detect a past strong bottleneck. The deviation from the expected frequencies of haplotypes is so large that even at this low sample size this will be apparent. Power did increase with increasing sample size, but for the detection of moderate bottlenecks very large sample sizes are necessary, if possible at all. The reason for this is clearly that the larger the deviation from expected, the more severe the bottleneck, and deviations that are only minor need very large sample sizes to be detected (a standard result in statistics). Since the pattern obtained here is a result of drift, moderate bottlenecks will be less affected. Likewise, recurrent immigration from a large source population will restore equilibrium, again a standard result from theoretical population genetics. Turning things around, the results presented here show that if there has been a strong bottleneck, the test proposed here, $\operatorname{Var}(S)$, is very effective in detecting it, even at low sample sizes.

The fact that power increased only marginally with increasing sample size is not particularly surprising and has been observed in other studies as well (eg, Wall, 1999). One reason in the current study is probably due to the rapid decline in sampling variance of $\theta$ with sample size observed by $\mathrm{Fu}$ and $\mathrm{Li}$ (1993b). An additional increase in power will probably be gained by the sampling of additional independent genes, since this will reduce the stochastic variance induced by the variance of evolutionary histories (Wakeley, 1996). This is the background of the method designed by Galtier et al (2000, see below).

One point that is not covered here is the time scale for which this test is effective. The simulations stopped when population size reached 1000 individuals, which in the slow $(r=0.009)$ increase case happened at around 200 generations, and at about 50 generations in the fast $(r=0.09)$ increase case. As time passes and population size increases mutations will accumulate; however, the time until an equilibrium situation is restored after a strong bottleneck will be very long since the rate of accumulation of neutral mutations equals the mutation rate (Kimura, 1968), which often is in the order of $10^{-6}$. Thus, the deviations from equilibrium haplotype frequencies after a bottleneck are likely to persist over considerable time periods, and the test proposed may well be suitable for testing possible bottlenecks in relation to Pleistocene glaciations and subsequent colonisations. This is supported by the data presented for the greenfinch (Table 1).

The fact that bottlenecks often result in distributions that are far from the expected has previously been used 
for the design of tests for bottlenecks using diploid data such as microsatellites (Cornuet and Luikart, 1996; Kimmel et al, 1998; Luikart and England, 1999; Reich et al, 1999; Garza and Williamson, 2001). This method is the first time that this possibility has been investigated for haploid DNA sequences. Recently, a different approach was described by Galtier et al (2000). They use a maximum likelihood approach that makes a more efficient use of the data allowing for the estimation of time since the bottleneck and the size of the bottleneck. However, for the one-locus data set (comparable to the one used here), the power was only $23 \%$ for a recent bottleneck (based on only 100 simulations and eight sampled individuals), but increased substantially (84\%) if three loci were used. Thus, given data from only one locus such as mtDNA control region data, this method seems less efficient than $\operatorname{Var}(S)$ for the detection of a bottleneck, although $\operatorname{Var}(S)$ cannot be used for estimating time and size of the bottleneck. Too much emphasis on these differences should probably be avoided since the conditions under which the simulations were performed differ in many ways.

The method used here assumes that the original population was large, panmictic and following an infinite-sites mutational model. Clearly, this will be violated in many cases - the original population may show various levels of substructure, nonrandom mating and may have gone through drastic changes in population size itself in earlier times. These factors will tend to decrease $\theta$, and thus lower the power to detect a bottleneck since $\theta$ is the only parameter estimated from the data.

\section{Acknowledgements}

I thank Jon Stone, John Brookfield and the reviewers for valuable comments on the manuscript. This work was supported by grants from the Swedish Natural Science Research Council.

\section{References}

Cornuet JM, Luikart G (1996). Description and power analysis of two tests for detecting recent population bottlenecks from allele frequency data. Genetics 144: 2001-2014.

Ellegren $H$ (2000). Microsatellite mutations in the germline: implications for evolutionary inference. Trends Genet 16: 552-559.
Elton CS (1958). The Ecology of Invasions by Animals and Plants. Methuen: London.

Fu Y-X, LI W-H (1993a). Statistical test of neutrality of mutations. Genetics 133: 693-709.

Fu Y-X, Li W-H (1993b). Maximum likelihood estimation of population parameters. Genetics 134: 1261-1270.

Galtier N, Depaulis F, Barton NH (2000). Detecting bottlenecks and selective sweeps from DNA sequence polymorphism. Genetics 155: 981-987.

Garza JC, Williamson EG (2001). Detection of reduction in population size using data from microsatellite loci. Mol Ecol 10: 305-318.

Hudson RR (1990). Gene genealogies and the coalescent process. Oxf Surv Evol Biol 1: 1-44.

Kimmel M, Chakraborty R, King JP, Bamshad M, Watkins WS, Jorde LB (1998). Signatures of population expansion in microsatellite repeat data. Genetics 148: 1921-1930.

Kimura M (1968). Evolutionary rate at the molecular level. Nature 217: 624-626.

Luikart G, England PR (1999). Statistical analysis of microsatellite DNA data. Trends Ecol Evol 14: 253-256.

Merilä J, Björklund M, Baker AJ (1997). Historical demography and present day population structure of the Greenfinch, Carduelis chloris - an analysis of mtDNA control-region sequences. Evolution 51: 946-956

Nei M, Maruyama T, Chakraborty R (1975). The bottleneck effect and genetic variability in populations. Evolution 29: $1-10$.

Pianka ER (1978). Evolutionary Ecology. Harper \& Row: New York.

Reich DR, Feldman MW, Goldstein DB (1999). Statistical properties of two tests that use multilocus data sets to detect population expansions. Mol Biol Evol 16: 453-466.

Rogers AR (1995). Genetic evidence for a Pleistocene population expansion. Evolution 49: 608-615.

Rogers AR, Harpending H (1992). Genetic evidence for a Pleistocene population expansion. Mol Biol Evol 9: 552-569.

Schneider S, Excoffier L (1999). Estimation of past demographic parameters from the distribution of pairwise differences when mutation rates vary among sites: application to human mitochondrial DNA. Genetics 152: 1079-1089.

Tajima F (1989a). Statistical methods for testing the neutral mutation hypothesis by DNA polymorphism. Genetics 123: 585-595.

Tajima F (1989b). The effect of change in population size on DNA polymorphism. Genetics 123: 597-601.

Wakeley J (1996). The variance of pairwise nucleotide differences in two populations with migration. Theor Popul Biol 49: 39-57.

Wall JD (1999). Recombination and the power of statistical tests of neutrality. Genet Res Camb 74: 65-79.

Watterson GA (1975). On the number of segregating sites in genetical models without recombination. Genetics 7: 256-276. 